\title{
Dor de dentes e fatores associados entre adolescentes de um município de grande porte populacional no Nordeste brasileiro
}

\author{
Tooth pain and associated factors among adolescents of a large \\ population municipality in Northeastern Brazil
}

\author{
Andreza Cristina de Lima Targino Massoni (https://orcid.org/0000-0002-3332-8315) ${ }^{1}$ \\ Érika Porto (https://orcid.org/0000-0002-0287-6933) ${ }^{1}$ \\ Luizy Raquel Barbosa Oliveira Ferreira (https://orcid.org/0000-0002-3810-6463) ${ }^{1}$ \\ Monalisa da Nóbrega Cesarino Gomes (https://orcid.org/0000-0001-7679-997X) ${ }^{1}$ \\ Ana Flávia Granville-Garcia (https://orcid.org/0000-0002-6054-8372) ${ }^{1}$ \\ Sérgio D'Avila (https://orcid.org/0000-0002-7836-896X) ${ }^{1}$
}

${ }^{1}$ Departamento de Odontologia, Universidade Estadual da Paraíba. Rua Juvêncio Arruda s/n, Bodoncogó. 58109-790 Campina Grande PB Brasil. andrezatargino@gmail.com

\begin{abstract}
The aim of this study was to describe and analyze history of toothache and associated factors among adolescent students enrolled in public schools of a city in Northeastern Brazil. An observational, descriptive, quantitative and cross-sectional study was conducted through the application of 4 questionnaires with 458 adolescents. By bivariate analysis, the prevalence of toothache in life was higher among younger adolescents (76.9\%; $p=0.004)$, who have visited the dentist at least once $(74.8 \% ; p=0.001)$ and who reported fear $(74.9 \%$; $p=0.006)$. The occurrence of dental pain in the last 6 months was low, where male $(77.4 \% ; p=0,001)$ and older adolescents $(73.1 \% ; p=0.031)$ reported no occurrence of pain. Through multivariate analysis, the highest prevalence of dental pain in life remained among younger adolescents (76.9\%; $p=$ $0.003)$ and who reported dental fear $(74.9 \%$; $p=$ 0.006). The prevalence of toothache in the last 6 months remained low through multivariate analysis, among male and older adolescents. It could be concluded that the prevalence of toothache in life was higher than in the last 6 months, showing significant association with gender, age, visit to the dentist and dental fear.
\end{abstract}

Key words Adolescents, Oral health, Fear, Anxiety, Toothache
Resumo O objetivo deste estudo foi descrever e analisar o histórico de dor de dente e seus fatores associados, entre adolescentes de escolas públicas de um município do Nordeste do Brasil. Realizou-se uma pesquisa observacional, descritiva, quantitativa e transversal, através da aplicação de quatro questionários junto a 458 adolescentes. Pela análise bivariada, a prevalência de dor de dente na vida foi alta entre os adolescentes de menor idade (76,9\%; $p=0,004)$, que visitaram o CirurgiãoDentista alguma vez $(74,8 \% ; p=0,001)$ e que relataram medo frente ao atendimento odontológico (74,9\%; $p=0,006)$. Todavia, nos últimos 6 meses, a prevalência de dor de dente foi baixa, na qual os adolescentes do sexo masculino (77,4\%; $p<0,001)$ $e$ de maior idade $(73,1 \% ; p=0,031)$ relataram justamente não terem sentido dor. Quando da análise multivariada, a maior prevalência de dor de dente na vida se manteve entre os adolescentes de menor idade $(76,9 \% ; p=0,003)$ e que relataram medo $(74,9 \% ; p=0,006)$. A prevalência de dor de dente nos últimos 6 meses se manteve baixa na análise multivariada, entre os adolescentes do sexo masculino e de maior idade. Conclui-se que a prevalencia de dor de dente na vida foi maior do que nos últimos 6 meses, havendo associação significativa com os fatores: sexo, idade, visita ao Cirurgião-Dentista e sensação de medo.

Palavras-chave Adolescente, Saúde bucal, Medo, Ansiedade, Odontalgia 


\section{Introdução}

A dor de dente constitui um tema relevante, pois nos dias atuais ainda é apontada como um dos principais problemas que levam as pessoas a procurar atendimento odontológico, tornando-se assim, um fator impactante na saúde pública ${ }^{1,2}$. Isto acontece, porque sua amplitude é suficientemente capaz de gerar situações indesejáveis e negativas na vida dos indivíduos, como dificuldades para comer e dormir, bem como a diminuição da produtividade no trabalho e na escola ${ }^{3}$. Profissionais envolvidos com os cuidados com a saúde reafirmam a importância deste assunto, ressaltando que a dor de dente demanda a capacidade de um adequado diagnóstico e técnicas de manuseio na Odontologia .

A dor é uma experiência por meio da qual a maioria das afecções se manifesta, podendo se expressar de diferentes formas e em distintas populações. De acordo com o Comitê de Taxonomia da International Associaton for the Study of Pain, um dos modelos explicativos da dor a define como uma experiência sensorial e emocional desagradável, decorrente ou descrita em termos de lesões teciduais ${ }^{5}$. $\mathrm{Na}$ área da Odontologia, a dor de dente consiste em um dos principais sintomas de sua prática, apresentando etiologia em componentes neurológicos, fisiológicos e psicológicos, além de ser caracterizada como proveniente ou originária dos tecidos inervados do dente, ou em estruturas adjacentes ${ }^{6}$. Destaca-se que fatores psicológicos podem apresentar influência na percepção que os indivíduos têm do atendimento odontológico, e assim, a ansiedade, o estado de atenção e as emoções podem levar a superestimar a dor que sentiram ${ }^{7}$.

Neste contexto, Lucas et al. ${ }^{8}$ fizeram importantes reflexões sobre o que, realmente, seria a dor. Destacando que qualquer indivíduo pode se reportar à sua experiência pessoal e dizer que sabe o que é a dor, ainda que a busca de palavras para expressar esta sensação seja difícil. Os autores ressaltaram que muitas definições podem ser dadas, mas nunca abarcará a dimensão do que é este problema, isto porque a dor é uma experiência subjetiva, privada, e qualquer informação sobre ela somente a pessoa que a sente pode expressá-la. Às vezes pode-se deduzir pelo comportamento, postura e expressões faciais que o individuo está sentindo dor e por estas indicações até pode-se localizá-la, mas os demais aspectos a seu respeito só o indivíduo pode informar.

Apesar de acometer indivíduos de diferentes faixas etárias, tem-se percebido que esta se evidencia no grupo dos adolescentes, ao qual, segun- do a Organização Mundial de Saúde, se enquadra na faixa etária entre 10 e 19 anos. Considerando o último levantamento epidemiológico a nível nacional, o SB Brasil ${ }^{10}, 24,6 \%$ dos adolescentes brasileiros procuraram atendimento odontológico devido à dor de dente. Aspecto que pode ser justificado pelo fato de ser a adolescência uma fase onde existe a busca pelo equilíbrio físico, psíquico e social, fazendo com que na maioria das vezes esses indivíduos apresentem comportamentos extremos e sejam de difícil adaptação/ aceitação, mostrando-se às vezes negligentes e omissos nos cuidados com a saúde bucal ${ }^{9}$.

Estudos têm destacado relações estreitas entre a dor de dente e determinados fatores, dentre eles: $\mathrm{o}$ acesso aos serviços de saúde bucal, a ansiedade e o medo odontológico ${ }^{11-13}$.

Neste contexto, observa-se que a dor de dente pode estar intimamente ligada às condições de acesso da população aos serviços de saúde bu$\mathrm{cal}^{14,15}$. Assim, a persistência das dificuldades no acesso aos serviços odontológicos por expressiva parcela da população brasileira, impede a prevenção ou tratamento dos agravos em saúde bucal $^{16,17}$, acarretando na exacerbação de sintomatologia dolorosa de origem dentária e, consequentemente, afetando, inclusive, a qualidade de vida dos adolescentes ${ }^{18}$.

Outro aspecto importante na relação entre dor de dente e atendimento odontológico diz respeito às sensações de medo e ansiedade, as quais são capazes de tornar os adolescentes menos propensos a procurar o serviço odontológico receosos de sentirem dor e pela grande ansiedade ${ }^{2,19}$.

Estes agravos podem ser considerados indicadores de saúde, se observados os impactos psicossociais que os mesmos ocasionam, como, por exemplo, o fato de ser um sintoma motivador para que o paciente procure ou retarde ainda mais o tratamento odontológico ${ }^{20,21}$.

Se tem observado que a maioria dos trabalhos que avaliam a dor de dente, o fazem de maneira isolada, ou seja, verificam, principalmente, a percepção dos indivíduos sobre a sintomatologia. Assim, torna-se relevante o desenvolvimento desse estudo; que busca verificar, justamente, a auto percepção da dor de dente associada a fatores como acesso aos serviços de saúde bucal, medo e ansiedade. Acrescenta-se a relevância do tema e a necessidade de quantificá-lo, de modo que estratégias no campo da saúde coletiva sejam lançadas no sentido de melhorar as ações educativas e a abordagem desses indivíduos.

Nesta perspectiva, este artigo tem como objetivo descrever e analisar o histórico de dor de dente e seus fatores associados, entre adolescen- 
tes de escolas públicas, de um município de grande porte populacional, do Nordeste do Brasil.

\section{Métodos}

Tratou-se de uma pesquisa observacional, descritiva, quantitativa, do tipo transversal, realizada nas escolas públicas de Campina Grande, um município de grande porte populacional, com Índice de Desenvolvimento Humano (IDH) igual a 0,720 , localizado na mesorregião do Agreste Paraibano, a $112 \mathrm{~km}$ da Capital do Estado, João Pessoa, Nordeste do Brasil ${ }^{22}$.

O universo desse estudo foi constituído por 29.838 adolescentes de 10 a 19 anos, matriculados em escolas públicas. Para determinação do tamanho amostral, utilizou-se fórmula para calcular populações finitas, e se considerou: população finita de 29.838 adolescentes, erro aceitável de 5,0\%, nível de confiança de $95 \%$ e prevalência de $50,0 \%$ (para valor desconhecido do fenômeno). Desta forma, obteve-se uma amostra de 380 alunos, a qual foi acrescentada $20 \%$ para suprir possíveis perdas (76 adolescentes) ${ }^{23}$. Assim, a amostra final pode ser constituída por um número de participantes entre 380 e 456 voluntários, distribuídos de forma estratificada, nos 8 Distritos Sanitários do município.

Quanto à coleta de dados, foram aplicados quatro questionários junto aos adolescentes, os quais eram relacionados: à dor de dente ${ }^{24}$, ao acesso aos serviços de saúde ${ }^{25}$, à avaliação do medo odontológico ${ }^{26} \mathrm{e}$ à avaliação da ansiedade den$\mathrm{tal}^{27}$. Acrescenta-se que foram adicionadas variáveis ligadas ao sexo e a idade dos participantes.

$\mathrm{O}$ questionário relacionado à dor de dente $\mathrm{e}^{24}$ foi utilizado com a finalidade de verificar a presença ou ausência deste agravo entre os adolescentes, em dois momentos: alguma vez na vida e nos últimos seis meses. Utilizou-se uma escala crescente para avaliar a duração da dor de dente. A intensidade da dor de dente foi avaliada por uma escala verbal ${ }^{28}$.

O questionário de acesso aos serviços de saúde objetivou avaliar a entrada nos serviços de saúde bucal por parte dos adolescentes e a procura por atendimento odontológico. Este questionário foi baseado no inquérito adotado na Pesquisa Nacional por Amostragem de Domicílios ${ }^{25}$.

O medo odontológico foi avaliado utilizando-se o Dental Fear Survey ${ }^{26}$, validado para a língua portuguesa por Cesar et al. ${ }^{29}$ e Costa e Moraes $^{30}$. Este instrumento é uma escala tipo likert de 5 pontos (com cinco opções de resposta) com
20 itens que, na versão original, mede 3 fatores: a fuga ao tratamento odontológico, as manifestações fisiológicas do medo e o medo provocado. Após primeira observação dos dados, os participantes foram classificados em com ou sem medo odontológico.

O Modified Dental Anxiety Scale foi utilizado para identificar a ansiedade dental ${ }^{27,31}$. A escala é composta de cinco questões com cinco alternativas de respostas, sendo que se atribui valor 1 à alternativa correspondente a de menor grau de ansiedade e valor 5 , à de maior grau. O mínimo escore possível é 5 (sem ansiedade) e o máximo, 25 (extrema ansiedade). Para este estudo, os indivíduos foram categorizados de acordo com a presença ou ausência de ansiedade.

É válido ressaltar que os questionários referentes às sensações de ansiedade e medo foram respondidos apenas pelos adolescentes que já haviam visitado o Cirurgião-Dentista em algum momento da vida, inviabilizando assim, que adolescentes que nunca utilizaram os serviços de saúde bucal os respondessem.

Para a análise estatística, os dados obtidos foram categorizados em dois grupos segundo a idade (10 a 14 anos, 15 a 19 anos) e o sexo (masculino e feminino). Os dados foram processados no programa estatístico SPSS (Statistical Package for Social Science) versão 20.0. Foi realizada análise bivariada e multivariada através da Regressão de Poisson para verificar a relação entre o histórico da dor de dente na vida e nos últimos 6 meses e seus fatores associados (sexo, faixa etária, visita ao dentista alguma vez na vida, visita ao dentista nos últimos 6 meses, ansiedade e medo) entre os adolescentes. As variáveis com um valor de $\mathrm{p}<0,20$ no modelo bivariado foram inseridas no modelo de regressão multivariado por meio de um procedimento backward stepwise. No modelo final foram consideradas como associadas às variáveis com um valor de $\mathrm{p}<0,05$. Em todos os testes foram adotados níveis de significância de 5\%.

Esta pesquisa foi submetida à apreciação do Comitê de Ética da Universidade Estadual da Paraíba, obtendo aprovação. O mesmo seguiu os princípios éticos propostos na Resolução 466/2012 do Conselho Nacional de Saúde, sendo garantido o sigilo dos dados referentes aos participantes.

\section{Resultados}

Ao término da coleta de dados, 458 adolescentes participaram do presente estudo, obtendo uma 
taxa de resposta de mais de 100,0\%. De modo geral, a prevalência de dor de dente na vida entre os adolescentes foi de $65,7 \%$; enquanto nos últimos 6 meses foi de $28,6 \%$. Os adolescentes relataram que a sensação de dor durou um período muito pequeno $(32,2 \%)$. Sendo esta, principalmente, desconfortável (40,2\%) (Tabela 1).

Ao analisar os dados acerca do histórico da dor de dente na vida e seus fatores associados, como demonstrado na Tabela 2, consideraramse as respostas de 437 adolescentes, aqui então, obtendo-se uma taxa de resposta de 95,4\%. Um total de 21 adolescentes não participou desta parte da análise, por responderem não lembro/não sei sobre o histórico de dor de dente na vida.

Percebe-se que a ocorrência de dor de dente na vida apresenta uma grande prevalência entre os adolescentes do sexo feminino $(73,2 \%)$, como também entre os adolescentes de menor faixa idade (76,9\%). Entre essas duas variáveis analisadas, apenas a variável faixa etária obteve valores com diferença estatisticamente significativa, em ambas as análises: bivariada $(\mathrm{p}=0,004)$ e multivariada $(\mathrm{p}=0,003)$ (Tabela 2).

Tabela 1. Frequência da prevalência, a intensidade e as características da dor de dente entre os adolescentes de escolas públicas.

\begin{tabular}{lr}
\hline \multicolumn{1}{c}{ Variáveis } & Frequência (\%) \\
\hline Dor de dente na vida & $136(29,7)$ \\
Não & $301(65,7)$ \\
Sim & $21(4,6)$ \\
Não lembra & \\
Dor de dente nos últimos 6 meses & \\
Não & $299(65,3)$ \\
Sim & $131(28,6)$ \\
Não lembra & $28(6,1)$ \\
Duração da dor & \\
Tempo muito pequeno & $97(32,2)$ \\
O dia inteiro & $39(13,1)$ \\
A noite inteira & $51(16,9)$ \\
Mais que um dia & $38(12,6)$ \\
Não lembra & $76(25,2)$ \\
Intensidade da dor & \\
Leve & $55(18,3)$ \\
Desconfortável & $121(40,2)$ \\
Estressante & $42(14,0)$ \\
Horrível & $68(22,6)$ \\
Intolerável & $15(5,0)$ \\
\hline
\end{tabular}

Fonte: elaboração própria.
É possível observar também dados relacionados ao acesso dos adolescentes aos serviços de saúde bucal, onde a ocorrência de dor de dente na vida aconteceu com maior prevalência entre os adolescentes que visitaram o Cirurgião-Dentista alguma vez na vida $(72,1 \%)$, com diferença estatisticamente significativa na análise bivariada $(\mathrm{p}=0,001)$, porém, na análise multivariada os valores não se mantiveram. Quanto a variável visita ao dentista nos últimos 6 meses, ela teve uma associação na análise bivariada com a variável dor de dente ( $\mathrm{p}=0,011)$, no entanto essa associação foi perdida na análise multivariada $(p>0,05)$ (Tabela 2).

Dentre os adolescentes com histórico de dor de dente na vida, $74,3 \%$ relataram se sentirem ansiosos frente ao atendimento odontológico, porém, sem diferença estatisticamente significativa. Todavia, estes relataram sentir medo $(74,9 \%)$ frente ao atendimento odontológico, com diferença estatisticamente significativa para as análises bivariada $(\mathrm{p}=0,006)$ e multivariada $(\mathrm{p}=$ 0,006) (Tabela 2).

Na Tabela 3 se verificam dados sobre o histórico de dor de dente apenas nos últimos 6 meses e seus fatores associados. 430 adolescentes responderam aos questionários referentes à dor de dente nos últimos seis meses (taxa de resposta de $93,8 \%$ ). Um total de 28 adolescentes não participou desta investigação, por responderem não lembro/não sei sobre o histórico de dor de dente nos últimos seis meses ou por terem se recusado a participar do estudo.

Percebe-se que a ocorrência de dor de dente nos últimos 6 meses apresentou menor frequência para as variáveis sexo e faixa etária quando comparados ao histórico de dor de dente na vida. Ainda assim, se manteve o padrão de adolescentes do sexo feminino mais jovens, com maior relato da dor de dente (Tabela 3 ).

É possível observar também na Tabela 3, dados relacionados ao acesso dos adolescentes aos serviços de saúde bucal, situação na qual a ocorrência de dor de dente nos últimos 6 meses apresentou baixa frequência. Enquanto $31,5 \%$ dos adolescentes que visitaram o Cirurgião-Dentista alguma vez na vida sentiram dor de dente; $33,8 \%$ daqueles adolescentes que visitaram o CirurgiãoDentista nos últimos 6 meses relataram sentir dor de dente. Ambos demonstraram relação estatisticamente significativa no modelo bivariado, mas estas relações não se mantiveram no modelo multivariado.

Dentre os adolescentes com histórico de dor de dente nos últimos 6 meses, 33,0\% relataram se 
Tabela 2. Análise bivariada e multivariada através da Regressão de Poisson em relação ao histórico de dor de dente e seus fatores associados entre adolescentes de escolas públicas.

\begin{tabular}{|c|c|c|c|c|c|c|}
\hline \multirow{2}{*}{ Variável } & \multicolumn{2}{|c|}{$\begin{array}{c}\text { Histórico de dor de } \\
\text { dente na vida }\end{array}$} & \multirow{2}{*}{\multicolumn{2}{|c|}{$\begin{array}{c}\text { Bivariada } \\
\text { RP Não ajustada }{ }^{\star *} \\
\text { p-valor }(95 \% \text { IC) }\end{array}$}} & \multirow{2}{*}{\multicolumn{2}{|c|}{$\begin{array}{c}\text { Multivariada } \\
\text { RP Ajustada } \dagger \\
\text { p-valor (95\% IC) }\end{array}$}} \\
\hline & $\begin{array}{c}\text { Sim } \\
\mathbf{n}(\%)\end{array}$ & $\begin{array}{c}\text { Não } \\
\mathbf{n}(\%) \\
\end{array}$ & & & & \\
\hline \multicolumn{7}{|l|}{ Sexo } \\
\hline Feminino & $161(73.2)$ & $59(26.8)$ & 0.052 & $1.13(0.99-1.28)$ & 0.524 & $1.04(0.92-1.17)$ \\
\hline Masculino & $140(64.5)$ & $77(35.5)$ & & 1.00 & & 1.00 \\
\hline \multicolumn{7}{|l|}{ Faixa etária } \\
\hline 10 a 14 anos & $120(76.9)$ & $36(23.1)$ & 0.004 & $1.19(1.05-1.34)$ & 0.003 & $1.19(1.06-1.34)$ \\
\hline 15 a 19 anos & $181(64.4)$ & $100(35.6)$ & & 1.00 & & 1.00 \\
\hline \multicolumn{7}{|c|}{ Visita ao dentista alguma vez na vida } \\
\hline Sim & $287(72.1)$ & $111(27.9)$ & 0.001 & $2.00(1.31-3.06)$ & 0.306 & $1.22(0.96-1.78)$ \\
\hline Não & 14(35.9) & $25(64.1)$ & & 1.00 & & 1.00 \\
\hline \multicolumn{7}{|c|}{ Visita ao dentista nos últimos 6 meses } \\
\hline Sim & $157(74.8)$ & $53(25.2)$ & 0.011 & $1.17(1.03-1.33)$ & 0.401 & $1.05(0.93-1.19)$ \\
\hline Não & $144(63.4)$ & $83(36.6)$ & & 1.00 & & 1.00 \\
\hline \multicolumn{7}{|l|}{ Ansiedade } \\
\hline Com ansiedade & $263(74.3)$ & $91(25.7)$ & 0.029 & $1.36(1.03-1.79)$ & 0.408 & $1.12(0.85-1.48)$ \\
\hline Sem ansiedade & $24(54.5)$ & $20(45.5)$ & & 1.00 & & 1.00 \\
\hline \multicolumn{7}{|l|}{ Medo } \\
\hline Com medo & $271(74.9)$ & $91(25.1)$ & 0.006 & $1.68(1.16-2.43)$ & 0.006 & $1.65(1.15-2.37)$ \\
\hline Sem medo & $16(44.4)$ & $20(55.6)$ & & 1.00 & & 1.00 \\
\hline
\end{tabular}

sentirem ansiosos frente ao atendimento odontológico, sem diferença estatisticamente significante $(\mathrm{p}=0,103)$. Nesta mesma análise, em relação ao medo, 21,6\% relataram sentir medo frente ao atendimento odontológico, sem diferença estatisticamente significante.

\section{Discussão}

A dor de dente é um agravo de grande impacto na Saúde Pública, pois, dependendo de sua intensidade, pode refletir de forma negativa nas atividades cotidianas dos indivíduos acometidos, bem como na sociedade. Entre essas repercussões, destacam-se os custos econômicos decorrentes, sejam eles diretos relativos aos serviços de saúde, ou indiretos, referentes à ausência nas atividades laborais e acadêmicas, no caso de estudantes.

A prevalência de dor de dente entre os adolescentes foi alta, com a ressalva de que a maioria dos investigados relatou uma duração muito pequena da sensação dolorosa, sendo esta, considerada predominantemente como desconfortável. Aspecto que está de acordo com outros estudos ${ }^{13,19}$.

Corroborando com os achados de prevalência desse trabalho, Hack-Comunello et al. ${ }^{32}$ realizaram um estudo com 603 escolares de 12 anos na cidade de Joaçaba-SC e perceberam que $71,9 \%$ dos sujeitos da pesquisa relataram ter tido o sintoma pelo menos uma vez durante a vida. Dados semelhantes foram encontrados nos Estados de Sergipe ${ }^{9}$, no ano de 2011, com prevalência de $71 \%$ e São Paulo ${ }^{19}$, no ano de 2012, com prevalência de $71,29 \%$. Todavia, em outros estudos ${ }^{12,33}$ a prevalência de dor de dente nos últimos 6 meses foi inferior àquelas relatadas anteriormente, com $14,8 \%$ e $22,1 \%$, respectivamente, porém, semelhantes também aos dados contidos neste estudo. A menor prevalência pode ter sido decorrente das peculiaridades nas metodologias de cada estudo, como localização, tipo de oferta de serviço: público/privado e, principalmente, tempo da última visita ao Cirurgião-Dentista. Outra reflexão capaz de justificar essa disparidade é bastante lógica; ora, se a prevalência de dor de dente na vida 
Tabela 3. Análise bivariada e multivariada através da Regressão de Poisson em relação ao histórico de dor de dente nos últimos 6 meses e seus fatores associados entre adolescentes de escolas públicas.

\begin{tabular}{|c|c|c|c|c|c|c|}
\hline \multirow[t]{2}{*}{ Variável } & \multicolumn{2}{|c|}{$\begin{array}{c}\text { Histórico de dor de } \\
\text { dente nos últimos } 6 \\
\text { meses }\end{array}$} & \multicolumn{2}{|c|}{ Bivariada } & \multicolumn{2}{|c|}{ Multivariada } \\
\hline & $\begin{array}{c}\text { Sim } \\
\mathbf{n}(\%)\end{array}$ & $\begin{array}{l}\text { Não } \\
\text { n(\%) }\end{array}$ & \multicolumn{2}{|c|}{$\begin{array}{l}\text { RP Não ajustada }{ }^{\star *} \\
\text { p-valor }(95 \% \text { IC) }\end{array}$} & \multicolumn{2}{|c|}{$\begin{array}{c}\text { RP Ajustada } \dagger \\
\text { p-valor (95\% IC) }\end{array}$} \\
\hline Feminino & $82(38.5)$ & $131(61.5)$ & $<0.001$ & $1.70(1.26-2.29)$ & 0.002 & $1.63(1.20-2.22)$ \\
\hline Masculino & $49(22.6)$ & $168(77.4)$ & & 1.00 & & 1.00 \\
\hline \multicolumn{7}{|l|}{ Faixa etária } \\
\hline 10 a 14 anos & $57(36.8)$ & $98(63.2)$ & 0.031 & $1.36(1.02-1.81)$ & 0.037 & $1.35(1.01-1.80)$ \\
\hline 15 a 19 anos & $74(26.9)$ & $201(73.1)$ & & 1.00 & & 1.00 \\
\hline \multicolumn{7}{|c|}{ Visita ao dentista alguma vez na vida } \\
\hline Sim & $123(31.5)$ & $267(68.5)$ & 0.161 & $1.57(0.83-2.98)$ & 0.898 & $1.05(0.49-2.20)$ \\
\hline Não & $8(20.0)$ & $32(80.0)$ & & 1.00 & & 1.00 \\
\hline \multicolumn{7}{|c|}{ Visita ao dentista nos últimos 6 meses } \\
\hline Sim & $70(33.8)$ & $137(66.2)$ & 0.147 & $1.23(0.92-1.64)$ & 0.755 & $1.04(0.77-1.41)$ \\
\hline Não & $61(27.4)$ & $162(72.6)$ & & 1.00 & & 1.00 \\
\hline \multicolumn{7}{|l|}{ Ansiedade } \\
\hline Com ansiedade & $114(33.0)$ & $231(67.0)$ & 0.103 & $1.65(0.90-3.02)$ & 0.142 & $1.54(0.86-2.76)$ \\
\hline Sem ansiedade & $9(20.0)$ & $36(80.0)$ & & 1.00 & & 1.00 \\
\hline \multicolumn{7}{|l|}{ Medo } \\
\hline Com medo & $115(32.6)$ & $238(67.4)$ & 0.203 & $1.50(0.80-2.83)$ & - & - \\
\hline Sem medo & $8(21.6)$ & $29(78.4)$ & & 1.00 & - & - \\
\hline
\end{tabular}

foi maior do que nos últimos 6 meses, então, este fato se deu, pois as chances de um adolescente ter sentido dor de dente pelo menos uma vez ao longo de até 19 anos, é muito maior do que se comparada a apenas os últimos 6 meses.

Se considerando a associação entre dor de dente e a variável sexo, observa-se que a prevalência foi baixa, havendo diferença estatisticamente significativa entre os adolescentes do sexo masculino que não apresentaram dor de dente nos últimos 6 meses. É perceptível que não há consenso na literatura científica quanto à associação entre dor de dente e o sexo, ora havendo uma maior prevalência deste agravo no sexo masculino ${ }^{34}$, ora no sexo feminino ${ }^{5,19}$. Em relação à faixa etária, foi observada diferença estatisticamente significativa da seguinte forma: entre os adolescentes de menor idade que apresentaram dor de dente em algum momento da vida, corroborando com os achados de outros autores ${ }^{11,12,33}$; bem como, entre os adolescentes de maior idade, ao qual não sentiram dor de dente nos últimos 6 meses, concordando com outros estudos ${ }^{12,35}$.

É relevante refletir que a relação entre idade e dor de dente talvez não seja diretamente proporcional, uma vez que os adolescentes de menor idade relataram em sua maioria a dor. Este fato leva a análise de que os pré-adolescentes vivenciam uma série de mudanças e que estas, influenciam diretamente em seu comportamento e atitudes diárias, que podem ser percebidas pela carga acumulada de estresse e rebeldia, sendo mais adeptos aos cuidados estéticos com o corpo e cabelos, se comparadas à boca, gerando então a recusa nos cuidados diários com a higiene oral e, consequentemente, maiores casos de dor de dente no grupo de menor idade. Em adição, é interessante observar o estudo de Cabral et al. ${ }^{12}$, onde não houve casos de dor de dente entre indivíduos de maior idade. Os autores refletiram sobre a possibilidade de os mesmos começarem apresentar sensibilidade diminuída, devido à 
esclerose dos tecidos dentinários, bem como ao fato de serem mais independentes e procurarem mais o serviço odontológico.

$\mathrm{O}$ acesso aos serviços de saúde bucal é tido como um importante preditor de qualidade de vida, devendo então ser refletido considerando a presença da dor de dente ${ }^{5}$. Desta forma, é recomendada a visita periódica ao Cirurgião-Dentista, pelo menos uma vez ao ano, a fim de identificar precocemente problemas de saúde bucal.

Neste contexto, pôde-se perceber no presente estudo e em outros ${ }^{19,36}$ que a ocorrência de dor de dente na vida é diretamente proporcional ao tempo de visita dos adolescentes ao CirurgiãoDentista, com diferença estatisticamente significante. Ou seja, os adolescentes que mais sentiram dor de dente foram aqueles que procuraram o atendimento há mais tempo. Logo, questiona-se se estes adolescentes só procuravam o atendimento quando realmente não suportavam mais a dor? Tal reflexão pode ser válida desde que outros aspectos também sejam considerados, como a existência de barreiras que poderiam dificultar este atendimento e, consequentemente, postergar a ida, como: falta de profissional na Unidade Básica de Saúde da Família (UBSF) mais próxima, falta de material para execução de procedimentos, demanda exacerbada, entre outros fatores. Os impactos negativos causados pela dor de dente na vida dos indivíduos reforçam a necessidade de ações prioritárias na atual política de saúde bucal, ampliando o acesso da atenção àqueles com piores condições e saúde bucal.

Apesar dos avanços tecnológicos e científicos nos equipamentos e procedimentos odontológicos, os sentimentos de ansiedade e medo em relação às consultas com o Cirurgião-Dentista ainda permeiam a vida das pessoas.

Neste estudo, não houve diferença estatisticamente significante entre o histórico de dor de dente na vida, nos últimos 6 meses e a ansiedade. No entanto, é sabido que pelo simples fato de o adolescente sentir dor de dente e necessitar de algum procedimento mais invasivo, o indivíduo pode apresentar algum grau de ansiedade. Tais considerações foram evidenciadas em outros estudos ${ }^{11,37}$.

Quanto ao medo, houve diferença estatisticamente significativa entre este e o histórico de dor de dente alguma vez na vida, no entanto, não houve diferença para a variável dor de dente nos últimos 6 meses. Outros estudos também relataram a grande prevalência do medo ${ }^{13,38}$, cuja sensação ocasiona diversos problemas periódicos, pois quando o tratamento preventivo não é realizado, as doenças bucais progridem e tomam proporções que necessitam de tratamentos curativos mais invasivos e, consequentemente, mais dolorosos que despertam tal sensação, conjunta com a fuga do atendimento.

A dor de dente pode ser reconhecida como preditor, no que diz respeito ao acesso aos serviços odontológicos entre os adolescentes. Não só ela, mas também outros fatores, que variam desde problemas técnicos e de gestão, até sensações de medo e ansiedade, são condições que influenciam direta ou indiretamente no estado psicológico do paciente, gerando recusa e/ou adiamento do atendimento odontológico. É necessário que haja maior emprego de medidas preventivas; e de promoção em saúde bucal, através de ações coletivas que esclareçam a população quanto a sua importância; bem como a ampliação do acesso aos serviços de saúde, preferencialmente os de ordem pública, no intuito de trazer uma visão positiva dos indivíduos frente ao atendimento odontológico. Tais estratégias se tornam essenciais para a melhoria das condições bucais, diminuindo o sofrimento e, melhorando a qualidade de vida.

\section{Conclusão}

Os dados permitem concluir que a prevalência de dor de dente relatada pelos adolescentes foi alta.

Pela análise bivariada foi constatado que a ocorrência de dor de dente na vida foi mais prevalente entre os adolescentes de menor idade: 10 a 14 anos, que visitaram o Cirurgião-Dentista alguma vez na vida e que relataram sentir medo, frente ao atendimento odontológico. Se observou também que a ocorrência de dor de dente nos últimos 6 meses apresentou baixa frequência, onde os adolescentes do sexo masculino e de maior idade: 14 a 19 anos, relataram não terem sentido dor de dente nos últimos 6 meses.

A análise multivariada demostrou que houve maior prevalência de dor de dente na vida, entre os adolescentes de menor faixa etária e aqueles que relataram a sensação de medo frente ao atendimento odontológico. No entanto, a prevalência de dor de dente nos últimos 6 meses apresentou baixa frequência, onde os adolescentes do sexo masculino e de maior idade relataram não terem sentido dor de dente nos últimos 6 meses.

Os dados desse estudo permitem entender que a dor de dente ocasiona consequências na vida das pessoas, influenciando no bem-estar e na qualidade de vida. Permitem também alertar os sistemas de saúde, no sentido de que melhorias precisam ser executadas no campo da saúde pública, in- 
cluindo estratégias de educação em saúde, no sentido de diminuir as sensações de medo e ansiedade frente ao atendimento odontológico, como também a ampliação do acesso aos serviços de saúde.

\section{Colaboradores}

ACLT Massoni, participou da concepção do projeto de pesquisa, da análise estatística e da redação do artigo. E Porto e LRBO Ferreira participaram da concepção do projeto de pesquisa, da coleta de dados e da redação do artigo. MNC Gomes participou da análise estatística e da redação do artigo. AF Granville-Garcia e S D'Avila participaram da análise estatística e da redação do artigo. 


\section{Referências}

1. Bastos JLD, Gigante DP, Peres KG, Nedel FB. Determinação social da odontalgia em estudos epidemiológicos: revisão teórica e proposta de um modelo conceitual. Cien Saude Colet 2007; 12(6):1611-1621.

2. Noro LRA, Roncalli AG, Teixeira AKM. Toothache and social and economic conditions among adolescents in Northeastern Brazil. Cien Saude Colet 2014; 19(1):105-113.

3. Mota JNG, Wanderley FGC, Silva RA, Almeida TF Absenteísmo por causa odontológica: uma revisão de literatura relacionada à ausência no trabalho e à saúde bucal do trabalhador. RFO 2015; 20(2):264-270.

4. Conti PCR, Kogawa EM, Vedolin GM, Calderon PS, Silva ROF. Diagnóstico diferencial das dores dentais. Rev Dor 2006; 7(3):845-853.

5. Borges CM, Cascaes AM, Fischer TK, Boing AF, Peres MA, Peres KG. Dor nos dentes e gengivas e fatores associados em adolescentes brasileiros: análise do inquérito nacional de saúde bucal SB-Brasil 2002-2003. Cad Saude Publica 2008; 24(8):1825-1834.

6. Knackfuss AP, Costenaro RGS, Zanatta FB. Dor odontológica e indicadores de risco em jovens. RGO 2011; 59(2):185-191.

7. Loggia ML, Schweinhardt P, Villemure C, Bushnell MC. Effects of psychological state on pain perception in the dental environment. J Can Dent Assoc 2008, 74(7):651-656.

8. Lucas SD, Mattos FF, Melo JAC, Vasconcelos M, Abreu MHNG, Ferreira NE. Uso de metáforas para expressar a dor de dente: um estudo na área de antropologia da saúde. Cien Saude Colet 2014; 19(6):1933-1942.

9. Carvalho RWF, Santos CNA, Oliveira CCC, Gonçalves SRJ, Novais SMA, Pereira MAS. Aspectos psicossociais dos adolescentes de Aracaju (SE) relacionados à percepção de saúde bucal. Cien Saude Colet 2011; 16(1):1621-1628.

10. Brasil. Ministério da Saúde (MS). Projeto SB Brasil 2010 - Pesquisa Nacional de Saúde Bucal: resultados principais. Brasília: MS; 2011.

11. Ferreira LL, Brandão GAM, Garcia G, Costa LST, Ambrosano GMB, Possobon RF. Odontalgia associada a variáveis socioeconômicas, psicossociais e saúde bucal. Rev Dor 2012; 13(4):343-349.

12. Cabral ED, Alves GG, Souza GC. Pain during dental care in family health units of Caruaru city, state of Pernambuco. Rev Dor 2013; 14(2):100-5.

13. Monteiro AX. Relação do medo, dor, ansiedade e condições de saúde bucal com o acesso aos serviços de saúde bucal e qualidade de vida de adolescentes [tese]. Bauru: Faculdade de Odontologia de Bauru; 2013.

14. Guiotoku SK, Moysés ST, Moysés SJ, França BHS, Bisinelli JC. Iniquidades raciais em saúde bucal no Brasil. Rev Panam Salud Publica 2012; 31(2):135-141.

15. Fonseca LLV, Nehmy RMQ, Mota JAC. O valor social dos dentes e o acesso aos serviços odontológicos. Cien Saude Colet 2015; 20(10):3129-3138.

16. Bottan ER, Pelegrini FM, Stein JC, Farias MMAG, Araújo SM. Relação entre consulta odontológica e ansiedade ao tratamento odontológico: estudo com um grupo de adolescentes. RSBO 2008; 5(3):27-32.
17. Rocha RACP, Goes PS. Comparison of access to Oral Health Services between areas covered and not covered by the Family Health Program in Campina Grande, Paraíba State, Brazil. Cad Saude Publica 2008; 4(12):2871-2880.

18. Souza TM, Roncalli AG. Oral health in the Brazilian Family Health Program: a health care mode evaluation. Cad Saude Publica 2007; 23(11):2727-2739.

19. Carvalho FS. Medo, ansiedade e dor de dente em adolescentes: impacto na qualidade de vida, na saúde bucal e no acesso aos serviços de saúde [tese]. Bauru: Faculdade de Odontologia de Bauru; 2012.

20. Josgrilberg EB, Cordeiro RCL. Aspectos psicológicos do paciente infantil no atendimento de urgência. Odontologia clínico-científica 2005; 4(1):13-19.

21. Kanegane K, Penha SS, Borsatti MA, Rocha RG. Dental anxiety in an emergency dental service. Rev Saude Publica 2003; 37(6):786-792.

22. Brasil. Censo Demográfico. Índice de Desenvolvimento Humano Municipal. Rio de Janeiro: IBGE; 2012.

23. Antunes JLF, Peres MA. Fundamentos de Odontologia: Epidemiologia da Saúde Bucal. Rio de Janeiro: Ed. Guanabara Koogan; 2006.

24. Goes PSA, Watt RG, Hardy R, Sheiham A. Impacts of dental pain on daily activities of adolescents aged 1415 years and their families. Acta Odont Scand 2008; 66(1):7-12.

25. Instituto de Geografia e Estatística (IBGE). Pesquisa Nacional por Amostra de Domicílios. Acesso e utilização de serviços de saúde. Rio de Janeiro: IBGE; 1998.

26. Kleinknecht RA, Keplac RK, Alexander LD. Origins and characteristics of fear of dentistry. J Am Dent Assoc 1973; 86(4):842-848.

27. Corah NL, Gale EN. Iiig SJ. Assessment of a dental anxiety scale. J Am Den Assoc 1978; 97:816-819.

28. Melzak R, Katz J. The McGill questionnaire: appraisal and current status. In: Turk DC, Melzak, editors. The Handbook of Pain Assessment. New York: Guildford Press; 1992. p. 152-168.

29. Cesar J, Moraes AB, Milgron P, Kleinknecht RA. Cross validation of a Brazilian version of the dental fear survey. Community Dent Oral Epidemiol 1993; 21:148150.

30. Costa SM, Moraes ABA. Medo em odontologia: um estudo com escolares. Rev Bras Odontol 1994; 51(5):26-31.

31. Pereira LHMC, Ramos DLP, Crosato E. Ansiedade e dor em odontologia - enfoque psicofisiopatológico. Rev APCD 1995; 49:285-290.

32. Hack-Comunello SM, Michel-Crosato E, Biazevic MGH, Crosato E. Dor dental e condição sócio-econômica: um estudo censitário em escolares. Pesq Bras Odontoped Clin Integr 2008; 8(1):63-67.

33. Constante HM, Bastos JL, Peres KG, Peres MA. Socio-demographic and behavioural inequalities in the impact of dental pain among adults: a population-based study. Community Dent Oral Epidemiol 2012; 40(6):498-506. 
34. Bastos JLD, Nomura LH, Peres MA. Dental pain, socioeconomic status, and dental caries in young male adults from southern Brazil. Cad Saude Publica 2005; 21(5):1416-1423.

35. Villalobos-Rodelo JJ, Medina-Solís CE, Maupomé G, Lamadrid-Figueroa H, Casanova-Rosado AJ, Casanova-Rosado JF, Márquez-Corona ML. Dental needs and socioeconomic status associated with utilization of dental services in the presence of dental pain: a case-control study in children. J Orofac Pain 2010; 24(3):279-286.

36. Gibilini C, Esmeriz CEC, Volpato LF, Meneguim ZMAP, Silva DD, Sousa MLR. Acesso a serviços odontológicos e auto-percepção da saúde bucal em adolescentes, adultos e idosos. Arquivos em Odontologia 2010; 46(4):213-223.

37. Armfield JM, Stewart JF, Spencer AJ. The vicious cycle of dental fear: exploring the interplay between oral health, service utilization and dental fear. BMC Oral Health 2007; 7(1):1-15.

38. Bottan ER, Oglio JD, Araújo SM. Ansiedade ao tratamento odontológico em estudantes do ensino fundamental. Pesq Bras Odontoped Clin Integr 2007; 7(3):241-246

Artigo apresentado em 28/07/2017

Aprovado em 08/06/2018

Versão final apresentada em 10/06/2018 